\title{
ON A CONJECTURE OF G. D. MOSTOW AND THE STRUCTURE OF SOLVMANIFOLDS
}

\author{
BY L. AUSLANDER AND R. TOLIMIERI
}

Communicated by Nathan Jacobson, June 10, 1969

Introduction. Let $G$ be a connected solvable Lie group and let $\Gamma$ be a closed subgroup of $G$. Then the quotient manifold $G / \Gamma$ is called a solvmanifold. G. D. Mostow in a fundamental paper [6] proved

TheOREM 1. Let $G / C$ be a compact solvmanifold, let $N$ be the nil-radical of $G$, and let $\Gamma$ contain no nontrivial, connected subgroup normal in $G$. Then

(a) $N$ contains the identity component of $\Gamma$,

(b) $N / N \cap \Gamma$ is compact,

(c) $N \Gamma$, the group generated by $N$ and $\Gamma$ in $G$, is closed, in $G$.

Mostow has also conjectured the following:

Mostow Conjecture. A solvmanifold is a vector bundle over a compact solvmanifold.

In this paper we will announce results that yield a new proof of Theorem 1 and a proof of the Mostow Conjecture, as well as many of the known results on the structure of solvmanifolds as given in [1], [3] and [4] for instance. An outline of the proof of the Mostow Conjecture and the proof of Theorem 1 are given in $\$ 3$.

1. Definitions and resume of known facts. Let $N$ be a connected, simply connected nilpotent Lie group. A closed subgroup of $N$ will be called a $C N$ group. According to Malcev a $C N$ group $\Delta$ can be characterized as a torsion free nilpotent group such that if $\Delta_{0}$ is the identity component of $\Delta$ then $\Delta / \Delta_{0}$ is finitely generated. Further, if $\Delta$ is a $C N$ group there exists a unique connected nilpotent Lie group $\Delta_{R}$ such that $\Delta_{R} \supset \Delta$ and $\Delta_{R} / \Delta$ is compact. If $\Delta$ is a $C N$ group with $\Delta_{0}$ trivial we will call $\Delta$ an $F N$ group.

In [3] and [6] it was shown that a group $\Gamma$ is the fundamental group of a compact solvmanifold if and only if $\Gamma$ satisfies an exact sequence

$$
1 \rightarrow \Delta \rightarrow \Gamma \rightarrow Z \cdot \rightarrow 1
$$

where $\Delta$ is an $F N$ group and $Z^{*}$ denotes $s$ copies of the integers. Fundamental groups of compact solvmanifolds will be called $F S$ groups. If $\Delta$ in (1) is a $C N$ group we will call $\Gamma$ a $C S$ group. If $\Gamma$ is a $C S$ group satisfying the exact sequence (1) there is a unique group $\Gamma_{R}$ satisfying the exact diagram: 


$$
\begin{gathered}
1 \rightarrow \Delta \rightarrow \Gamma \rightarrow Z^{*} \rightarrow 1 \\
\downarrow \quad \downarrow \quad \downarrow \\
1 \rightarrow \Delta_{R} \rightarrow \Gamma_{R} \rightarrow Z^{*} \rightarrow 1
\end{gathered}
$$

As it is convenient, we will often identify a connected simple connected nilpotent Lie group with its Lie algebra by the exponential mapping.

Now let $G$ be a connected simply connected solvable Lie group. In [5] the semisimple splitting $S$ of $G$ was characterized as follows:

$$
S=T \cdot M=T \cdot G
$$

where $M$ is the simply connected nil-radical of $S$ and $T$ is an abelian group of semisimple automorphisms of $M$, where $S$ is generated by $T$ and $G$.

In [7], Wang showed that if $\Delta$ is an $F N$ group, then

$$
1 \rightarrow \Delta_{R} \rightarrow \Gamma_{R} \rightarrow Z^{8} \rightarrow 1
$$

has a semisimple splitting in the following sense: there exists an abelian group of automorphism of $\Gamma_{R}$ such that:

(1) $T \cdot \Gamma_{R}=T \cdot \Delta^{*}$ where $\Delta^{*}$ is a $C N$ group and $\Delta^{*} \supset \Delta_{R}$.

(2) $T$ acts as a semisimple group of automorphisms of $\Delta_{R}{ }^{*}$ such that $T\left(\Delta_{R}\right)=\Delta_{R}$ and $T$ induces the trivial action on $\Delta_{R}^{*} / \Delta_{R}$.

2. Main theorems.

TheOREM 2. Let $\Gamma$ be a CS group satisfying the exact sequence (1) and let $\Gamma_{R}$ satisfy diagram (2). There exists a closed subgroup $\Delta^{*}$ of $\Delta_{R}$ such that

(a) $\Delta^{*} \supset \Delta$ and $\Delta^{*} / \Delta$ is finite,

(b) $\Delta^{*}$ is normalized by $\Gamma$.

Further, if we let $\Gamma^{*}=\Gamma \Delta^{*}$, there exists a semisimple splitting $T \cdot \Gamma_{R}$ of $\Gamma_{R}$ such that

(c) $\Gamma^{*}$ and $\Delta^{*}$ are invariant under $T$.

Definition. A group $T \cdot \Gamma^{*}$ satisfying the conclusion of Theorem 2 is called a semisimple splitting of $\Gamma$.

Theorem 3. Let $\Gamma$ be a $C \cdot S$ group and let $\Gamma_{1}^{*}$ and $\Gamma_{2}^{*}$ be two semisimple splittings of $\Gamma$. Then $\Gamma_{1}^{*}$ and $\Gamma_{2}^{*}$ are commensurable. Further, any automorphism of $\Gamma$ has a unique extension to any semisimple splitting of $\Gamma$.

LEMma 4. Let $G$ be a connected, simple connected solvable Lie group and let $\Gamma$ be a closed subgroup of $G$. Then $\Gamma$ is a $C \cdot S$ group. 
TheOREM 5 (NIL-SHADOW). Let $G$ be a connected, simple connected solvable Lie group and let $\Gamma$ be a closed subgroup of $G$. Let $S$ be the semisimple splitting of $S$ and let $\Gamma_{S}=T_{\Gamma} \cdot \Gamma^{*}$ be a semisimple splitting of $\Gamma$. There exists an isomorphism $\eta: \Gamma_{S} \rightarrow S$ such that $\eta\left(\Gamma_{S}\right)$ is a closed subgroup of $S$ with $\eta(\Gamma)=\Gamma$. Further, there exists a semidirect product presentation of $S=T \cdot M$ such that $\eta\left(T_{\Gamma}\right) \subset T$ and if $\Gamma_{S}=T_{\Gamma} \cdot \Delta^{*}$ then $\Delta^{*} \subset M$ as a closed subgroup, where $\Delta^{*}$ is a $C N$ group.

3. Applications. In order to show the power of the nil-shadow theorem, we will assume it and prove Theorem 1 and outline a proof of the Mostow Conjecture.

Definition. Let $N$ be a connected, simply connected nilpotent Lie group and let $B$ be a group of automorphisms of $N$. We define an action of $B \cdot N$ on $N$, called the affine action, as follows:

Let $(b, n) \in B \cdot N$ and let $m \in N$. We define

$$
(b, n)(m)=b^{-1}(m) n
$$

and if $\xi \in B \cdot N$ we denote $a(\xi)$ as the affine action of $\xi$ on $N$.

We begin by stating, without proof, the following lemma.

LEMMA 6. Let $G$ be a connected, simply connected solvable Lie group and let $\Gamma$ be a closed subgroup of $G$. Let $S=T \cdot M$ be the semisimple splitting of $G$. Then if we consider $\Gamma \subset S$ acting on $M$ by the affine action then $M / a(\Gamma)$ is diffeomorphic to $S / \Gamma$.

Outline of Mostow's Conjecture. Let $\Gamma_{S}=T_{\Gamma} \cdot \Delta^{*}$ be a semisimple splitting of $\Gamma$ with $\Delta^{*}$ in $M$ and $T_{\Gamma} \subset T$. Then $\Delta_{R}^{*}$ is a subgroup of $M$ which is invariant under $\Gamma$ and $\Delta_{R}^{*} / a(\Gamma)$ is a compact solvmanifold. Let $V$ be a vector space such that $M=V \oplus \Delta_{R}^{*}$ and such that $T_{\Gamma}(V)=V$. The existence of $V$ follows from the fact that $T_{\Gamma}$ leaves $\Delta_{R}^{*}$ invariant and that $T_{\mathrm{r}}$ acts semisimply on $M$. Hence every element of $M$ may be written uniquely as $v \cdot \delta$, where $\delta \in \Delta_{R}^{*}, v \in V$. Further if $\gamma=(t, n), n \in \Delta_{R}^{*}, t \in T_{\Gamma}$ we have

$$
(t, n) v \cdot \delta=t(v) \cdot t(\delta) n=v^{\prime} \delta^{\prime}
$$

where $v^{\prime} \in V$ and $\delta^{\prime} \in \Delta_{R}^{*}$. Thus we see that the images of the sets $V \cdot \delta, \delta \in \Delta_{R}^{*}$ gives a fiber bundle structure to $M / a(\Gamma)$ with $\Delta_{R}^{*} / a(\Gamma)$ as compact base space. It is easy to see that the action is linear and so that this is a vector bundle over the compact solvmanifold $\Delta_{R}^{*} / a(\Gamma)$.

Let us now prove Theorem 1. Assume now that all notation is as above and that in addition to this, $G / \Gamma$ is compact. Then by the proof of the Mostow Conjecture we have that $\Delta_{R}^{*}=M$ or $\Delta^{*}$ is a closed cocompact subgroup of $M$. Thus, if $\Delta_{0}^{*}$ denotes the identity component of $\Delta^{*}, \Delta_{0}^{*}$ is normal in $M$ and invariant under $T_{\Gamma}$. 
The following lemma is straightforward and its proof will be omitted.

LEMMA 7. Let all notation be as above. $\Gamma N / N$ is a discrete subgroup of $G / N$ if and only if $T_{\Gamma}$ is a discrete subgroup of $T$.

LEMmA 8. Let all notation be as above. If a $\left(M / \Delta_{0}^{*}\right)$ denotes the automorphism group of $M / \Delta_{0}^{f}$ then the natural homomorphism $\delta: T_{\Gamma}$ $\rightarrow a\left(M / \Delta_{0}^{\sharp}\right)$ has trivial kernel.

Proof. Let $t \in T_{\Gamma}$ be in the kernel of $\delta$. Then the range $X$ of $(t-I)$, where $I$ is the identity transformation, is in $\Delta_{0}^{\sharp}$. Since $T$ is abelian, $X$ is an invariant subspace of $M$ under $T$. Hence the ideal, $g(X)$, generated by $X$ in $M$ is in $\Delta_{0}^{*}$ and invariant under $T$. Thus $g(X)$ is an ideal in $\delta$ and so in $G$. This contradicts our hypothesis unless $X=0$ and the kernel of $\delta$ is trivial.

Proof of Theorem 1 . Since $\delta\left(T_{\Gamma}\right)$ preserves a discrete cocompact subgroup of $M / \Delta_{0}^{*}, \delta\left(T_{\Gamma}\right)$ is a discrete subgroup of $a\left(M / \Delta_{0}^{*}\right)$. Hence $T_{\Gamma}$ is a discrete subset of $a(M)$. Lemma 8 now applies to complete the proof of Theorem 1.

\section{REFERENCES}

1. L. Auslander, Solvable Lie groups acting on nilmanifolds, Amer. J. Math. 82 (1960), 653-660.

2. - Discrete uniform subgroups of solvable Lie groups, Trans. Amer. Math. Soc. 99 (1961), 398-402.

3. - Fundamental groups of compact solvmanifolds, Amer. J. Math. 82 (1960), 689-697.

4. L. Auslander and M. Auslander, Solvable Lie groups and locally Euclidean Riemann spaces, Proc. Amer. Math. Soc. 9 (1958), 933-941.

5. L. Auslander and J. Brezin, Almost algebraic Lie algebras, J. Algebra 8 (1968), 295-313.

6. G. D. Mostow, Factor spaces of solvable groups, Ann. of Math. (2) 60 (1954), $1-27$.

7. H. C. Wang, Discrete subgroups of solvable Lie groups. I, Ann. of Math. (2) 64 (1956), 1-19. 10036

Graduate Center, City University of New York, New York, New York 